\title{
Saber disciplinario en la formación inicial docente. El caso de los estudiantes de último año de carreras de Pedagogía en Educación Física y Deportes en Chile
}

\author{
Jorge Flández Valderrama \\ Universidad Austral de Chile. Instituto Ciencias de la Educación, Chile \\ jflandez@uach.cl \\ Marcelo González Orb \\ Universidad Metropolitana de Ciencias de la Educación. Departamento de Educación Física, \\ Chile \\ Rodrigo Vargas Vitoria \\ Universidad Catolica del Maule. Departamento de Ciencias de Actividad Física, \\ Chile \\ Edgardo Molina Sotomayor \\ Universidad Metropolitana de Ciencias de la Educación. Departamento de Educación Física, \\ Chile \\ Ana Beltrán González \\ Universidad de Antofagasta. Departamento de Educación, \\ Chile \\ Cristian Martínez Salazar \\ Universidad de la Frontera. Departamento de Educación Física, Deportes y Recreación, \\ Argentina \\ Héctor Trujillo Galindo \\ Universidad Central. Facultad de Ciencias de la Educación, \\ Argentina \\ Mariela Ferreira Urzúa \\ Universidad Metropolitana de Ciencias de la Educación. Departamento de Educación Física, \\ Chile \\ Roberto Leiva Contardo \\ Universidad Metropolitana de Ciencias de la Educación. Departamento de Educación Física, \\ Chile \\ Manuel Lobos González \\ Universidad Andrés Bello, \\ Chile \\ Mario Negrón Molina \\ Universidad de los Lagos. Departamento Ciencias de la Actividad Física, \\ Chile
}

Cita sugerida: Flández Valderrama, J., González Orb, M., Vargas Vitoria, R., Molina Sotomayor, E., Beltrán González, A., Martínez Salazar, C., Trujillo Galindo, H., Ferreira Urzúa, M., Leiva Contardo, R., Lobos González, M. y Negrón Molina, M. (2019). Saber disciplinario en la formación inicial docente. El caso de los estudiantes de último año de carreras de Pedagogía en Educación Física y Deportes en Chile. Educación Física y Ciencia, 21(4), e108. https://doi.org/10.24215/23142561e108 


\section{Resumen:}

Este trabajo, buscó determinar el desempeño de saberes disciplinarios de las y los estudiantes de último año de la Carrera de Educación Física y Deporte de Chile. Se describió y analizó el nivel de conocimientos disciplinarios como también diferencias significativas entre áreas, universidades, tipo de casas de estudios, género y dependencia escolar. Estudio descriptivo, observacional exploratorio, de carácter transversal asociadas a siete áreas de conocimientos disciplinarios vinculados a campos aplicativos y ejes temáticos propios del sistema educativo, propuestos en las Bases Curriculares Nacionales del Ministerio de Educación. El tipo de muestreo aplicado fue no probabilístico, por conveniencia, el cual consideró a seis universidades, contemplando un $\mathrm{n}=$ 214 estudiantes. Se empleó una prueba de selección múltiple, que consideró 70 ítems, centrado en conocimientos disciplinarios, sometido previamente a validación de contenido y de consistencia interna (alfa de Cronbach de .778), y pruebas de normalidad (Kolmogorov - Smirnov). Para determinar las diferencias entre distintas dimensiones, se utilizó estadística inferencial, ANOVA y prueba de Tuckey. Es posible determinar que los saberes disciplinarios de las y los estudiantes de último semestre, poseen un desempeño regular. Los resultados contribuyen a los planes de mejoras de las casas de estudios.

Palabras Clave: Saberes disciplinarios, Formación Inicial Docente, Carreras Educación Física.

\section{Abstract:}

Chile is facing a long and complex academic, social, and political discussion about the quality assurance of Initial Teacher training. The institutions that train Physical Education and Sports Teachers have diversified. Evaluation of the different dimensions of teacher training has created a network of relevant topics to be investigated. This work sought to evaluate the performance of disciplinary knowledge of senior students of the Physical Education and Sports Degree in Chile. The level of disciplinary knowledge was described and analysed, and differences between areas, universities, institution type, gender, and school dependency were also evaluated. This study was a descriptive, observational, exploratory, cross-sectional study associated with seven areas of disciplinary knowledge linked to applied fields and thematic axes of the educational system, proposed in the National Curricular Bases of the Ministry of Education. The sampling type was non-probabilistic, for convenience, which considered six universities, with a total number of 214 students. A multiple-choice test was used, with 70 questions, focused on disciplinary knowledge, which was previously subjected to content validation and internal consistency (Cronbach's alpha of .778), and normality tests (Kolmogorov Smirnov). To determine the differences between different dimensions, inferential statistics, ANOVA and Tuckey tests were used. We concluded that the disciplinary knowledge of students in their last semester was regular. These results will contribute to the improvement of curricular studies in the different institutes.

KEYWORDS: Disciplinary knowledge, Initial teacher training, Physical education degree.

\section{INTRODUCCIÓN}

A nivel mundial los sistemas de Educación Superior, están siendo sometidos a fuertes presiones para elevar la calidad de su enseñanza, hasta el punto que ésta se ha convertido en su prioridad estratégica (Cid, Alfonso, Andrés Pérez y Miguel Zabalza, 2009 y Hativa \& Goodyear, 2002). Existen distintos conceptos y perspectivas respecto a la Calidad y como tal, se declara como un concepto pluridimensional, continuo e integral y que necesita de una autoevaluación interna y otra externa, que permita satisfacer las necesidades internas, pero también las expectativas sociales(Machado,2018). En este sentido, establecer la calidad de la formación universitaria otorgada a las y los estudiantes ha sido una preocupación constante a través del tiempo (Ruffinelli,2014)

En el caso de la Formación Inicial Docente (FID), se trata de una tarea compleja, que requiere el aprendizaje de contenidos muy diversos e interrelacionados (Caena, 2011). Ante esta complejidad, se debe dotar a los estudiantes de los conocimientos y competencias necesarias para desarrollar con calidad su futuro despliegue como docente (Hortigüela, Pérez- Pueyo \& Rio-Fernández, 2016). En este contexto, si se tiene en cuenta la diversidad de contenidos asociados a la Educación Física, el conocimiento disciplinario, el conocimiento pedagógico y el conocimiento actitudinal, son tres elementos importantes para el desempeño de la futura labor profesional docente. Precisamente, es en este contexto, donde la evaluación del proceso formativo adquiere su relevancia y es la forma en que el docente recoge información sobre el aprendizaje de las y los estudiantes como también, de su propia enseñanza con el objetivo de mejorar el proceso de FID (López-Pastor \& Sicilia-Camacho, 2017). 
En este y otros ámbitos, Chile, se enfrenta a una larga y compleja discusión acerca del aseguramiento de la calidad de la FID en instituciones de Educación Superior (Ruffinelli, 2013). Al respecto, la calidad de la educación solo podrá ser mejorada si basa la enseñanza en profesores altamente calificados. Pare ello, es preciso tener en cuenta que la actualización, debe comenzar por la propia reflexión sobre su quehacer profesional (Pedraja-Rejas, Araneda-Guirriman, Rodríguez-Ponce \& Rodríguez-Ponce, 2012). En esa línea, la evaluación de las distintas dimensiones de formación de las carreras de Pregrado, son relevantes y por tal motivo en el último tiempo, se han desplegado esfuerzos importantes para mejorar la calidad y equidad de la Educación, planteándose como una de las prioridades, el fortalecimiento de la FID. Dentro de las propuestas de mejoramiento, se encuentran el Programa de Fortalecimiento de la Formación Inicial Docente (PFFID); La Beca Vocación de Profesor; la definición de Estándares de desempeño para la FID (Pedagógicos y Disciplinarios); la Acreditación obligatoria para las carreras de Pedagogía desde el año 2006, en su momento (2008), la implementación de la Evaluación Diagnóstica de la Evaluación Inicial Docente (Prueba INICIA) y actualmente, la Evaluación Nacional Diagnóstica (ENDFID). Todas ellas, un avance para enfrentar los desafíos de la calidad, esperables de la FID, que, en su momento, sumó también, las movilizaciones sociales por la Educación del año 2011, las cuales contribuyeron y en algunos casos, aceleraron los procesos de modificación de las actuales políticas en el área.

En ese contexto, desde el año 2014, se han venido impulsando reformas a la Educación con impacto y desafíos con foco en la Educación Superior, responsables de formar profesores, entre ellas, la Ley General de la Educación 20.470 (LGE), Ley de Educación Superior (21.091), Sistema de Desarrollo Profesional Docente (SDPD - Ley 20.903), Ley de Inclusión Escolar (Ley 20.845 y 20.609), Plan de Formación Ciudadana (Ley 20.911), todas ellas, con el propósito de poner en diálogo la formación universitaria con el nuevo contexto educativo y sociocultural del país. En este sentido, la pedagogía, se estableció como saber fundante de la FID y el saber profesional del mismo. Sin embargo, este proceso no puede estar desconectado del saber disciplinar y del conocimiento experiencial. A través de estos saberes, se construye la identidad profesional y el actuar docente que aparecen configurados por la práctica, el tiempo y la experiencia escolar y de vida, lo que permitirá tomar decisiones acerca del modo de aproximarse al currículum, reflexionar sobre la praxis, seleccionar y organizar sus saberes prácticos (Gaete,2008). Para ello, se requiere contar con formadores de formadores que tengan una adecuada preparación y experiencia práctica (Ávalos, 2005). De esta manera, mientras más amplio es el manejo pedagógico y disciplinar de un docente, mayores son las capacidades de incidir decididamente en el proceso de aprendizaje de sus propios estudiantes en formación (Bravo, Falk, González, Manzi \& Peirano, 2008).

En Chile, específicamente las instituciones que forman Profesores de Educación Física y Deportes, se han diversificado, implementando distintos proyectos y modelos curriculares, con diversos sustentos epistemológicos, enfoques, perfiles y temporalización, sin embargo, se carece de instrumentos de evaluación que permitan tener una apreciación respecto de los niveles de logro de conocimientos teóricos y aplicados que poseen los profesionales en formación en el campo disciplinario de la Educación Física. Estos procesos vinculados a la evaluación de la formación, permiten transitar desde la cultura de los test a la cultura de la evaluación (Desrosiers, Genet-Volet \& Godbout, 1997). Si bien es cierto, existen pocos estudios que correspondan a este tema, en Chile actualmente, se cuenta con algunos resultados de mediciones de conocimientos y habilidades de estudiantes de pedagogía, sin embargo, manifiestan falencias importantes no sólo en sus conocimientos disciplinarios, sino también pedagógicos (Sotomayor y Gysling, 2011; Ministerio de Educación [MINEDUC]- Centro de Perfeccionamiento, Experimentación e Investigaciones Pedagógicas [CPEIP], 2016-2017-2018).

En este particular escenario, el objetivo del estudio se orienta a determinar el desempeño de saberes disciplinarios de las y los estudiantes de último año de la Carrera de Educación Física y Deporte vinculadas a universidades del Consejo de Rectores de Chile. El trabajo describió y analizó el nivel de conocimientos disciplinarios como también, diferencias significativas entre áreas de conocimientos, universidades, tipo de 
casas de estudios, dependencia escolar y género. Esta última variable, en virtud de la actual política de equidad de género que se propicia en la Educación Superior chilena (Hidalgo \& Venegas, 2019) y que paralelamente, promueve la Organización de las Naciones Unidas para la Educación, la Ciencia y la Cultura (UNESCO, 2018) con el propósito de generar evidencias que permiten indagar trayectorias educativas y aportar a los cambios políticos y culturales que se necesita.

\section{Metodología}

Utiliza estudio descriptivo, observacional exploratorio, de carácter transversal, asociadas a siete áreas de conocimientos disciplinarios vinculados a campos aplicativos y ejes temticos propios del sistema educativo y propuestos en las Bases Curriculares Nacionales del Ministerio de Educación, entre ellas, Expresión Motriz, Didctica, Gestión, Entrenamiento, Deportes, Recreación, Salud y Primeros Auxilios.

El tipo de muestreo para seleccionar a las universidades fue de tipo no probabilístico, por conveniencia. Se tuvo acceso a información de seis universidades (4 Estatales y 2 Privadas con vocación y derecho público). Por cada universidad, se muestreó a los estudiantes de último año de las carreras de Pedagogía en Educación Física y Deporte, con un total de 214 estudiantes, la que se puede apreciar en la Tabla 1.

En la tabla 1, se presentan las principales características de la muestra:

Caracterización de la muestra por Universidad

TABLA 1

Caracterización de la muestra por Universidad

\begin{tabular}{lccccc}
\hline & \multicolumn{5}{c}{ GÉNERO } \\
\cline { 2 - 6 } & \multicolumn{2}{c}{ Hombre } & \multicolumn{2}{c}{ Mujer } & Total \\
\cline { 2 - 6 } UNIVERSIDAD & $\mathrm{N}$ & $\%$ & $\mathrm{n}$ & $\%$ & $\mathrm{n}$ \\
\hline \hline Universidad de Antofagasta & 11 & 55,0 & 9 & 45,0 & 20 \\
\hline UMCE & 18 & 51,4 & 17 & 48,6 & 35 \\
\hline UC Maule & 39 & 66,1 & 20 & 33,9 & 59 \\
\hline UFRO & 18 & 62,1 & 11 & 37,9 & 29 \\
\hline U Austral & 22 & 57,9 & 16 & 42,1 & 38 \\
\hline U Los Lagos & 25 & 75,8 & 8 & 24,2 & 33 \\
\hline Total & 133 & 62,1 & 81 & 37,9 & 214 \\
\hline \hline
\end{tabular}

Se utilizó como instrumento, una prueba de selección múltiple que consideró 90 ítems, centrado en conocimientos disciplinarios y las capacidades de aplicación de los mismos, sometido previamente a validación de contenido y de consistencia interna (alfa de Cronbach de .778), donde las pruebas de normalidad (Kolmogorov - Smirnov), revelaron distribución normal. El instrumento se encuentra publicado en revista indexada Retos, 37, 312-319, "Construcción y Validación de un Instrumento para Valorar la Calidad de la Formación de Profesores de Educación Física en Chile”, a través del cual se describen a cabalidad los protocolos utilizados. Se realizaron análisis estadísticos descriptivos de tendencia central y dispersión. Además, para determinar diferencias entre áreas de conocimientos disciplinarios, universidades, tipo de casas de estudios, género, dependencia escolar, se utilizó estadística inferencial, mediante ANOVA y prueba de Tuckey. Para todas las pruebas, se utilizó un nivel de significancia del 0.05. Los análisis fueron llevados a cabo mediante programa estadístico SPSS, versión 23. 
El estudio se realizó bajo estrictos criterios éticos y científicos. Tuvo la aprobación del Comité de Ética de la Universidad de Santiago de Chile, a través del informe No 334 del año 2017. Paralelamente, se contó con la autorización de las entidades universitarias implicadas en el estudio a través de sus respectivas Direcciones de Investigación como también, del consentimiento informado de cada sujeto participante.

\section{Resultados}

La tabla 2, devela que entre áreas de conocimientos y universidades existen diferencias significativas entre las medias (Valor $\mathrm{p}=0,00$ menor que nivel de significancia $\alpha=0,05$ ). La disciplina más descendida es Expresión y aquellas con mejor desempeño fueron Didáctica, Recreación, Entrenamiento y Deporte.

TABLA 2

Conocimientos disciplinarios entre Universidades

\begin{tabular}{|c|c|c|c|c|c|c|c|c|}
\hline Disciplina & U1 & $\mathrm{U} 2$ & U3 & U4 & U5 & U6 & Significancia & $\begin{array}{l}\text { Secuencia de mayor a } \\
\text { menor }\end{array}$ \\
\hline Expresión & 0,39 & 0,57 & 0,37 & 0,44 & 0,44 & 0,33 & $\mathrm{Si}$ & U1-U5 ; U4-U1- U3-U6 \\
\hline Didáctica & 0,66 & 0,68 & 0,59 & 0,57 & 0,55 & 0,58 & $\mathrm{Si}$ & U2-U1; U3-U6-U4-U5 \\
\hline Gestión & 0,53 & 0,53 & 0,5 & 0,57 & 0,55 & 0,51 & No & Similares \\
\hline Entrenamiento & 0,62 & 0,62 & 0,58 & 0,56 & 0,53 & 0,57 & No & Similares \\
\hline Deporte & 0,59 & 0,6 & 0,58 & 0,58 & 0,55 & 0,53 & No & Similares \\
\hline Recreación & 0,55 & 0,71 & 0,6 & 0,57 & 0,59 & 0,53 & $\mathrm{Si}$ & U2-U3-U5; U4-U1-U6 \\
\hline $\begin{array}{c}\text { Salud y } \\
\text { Primeros } \\
\text { Auxilios }\end{array}$ & 0,48 & 0,56 & 0,42 & 0,43 & 0,49 & 0,4 & $\mathrm{Si}$ & U2-U5-U1; U4- U3-U6 \\
\hline
\end{tabular}

Por su parte, en la tabla 3, se puede apreciar el dominio adquirido de conocimientos disciplinarios entre las universidades estatales y las universidades privadas con vocación y derecho público, donde sólo se observó diferencias significativas en la disciplina de Didáctica, en el que las Estatales tienen mejor evaluación. En general, se advierte simetría de conocimientos disciplinarios entre tipo de universidades 
TABLA 3

Conocimientos disciplinarios según tipo de Universidad

\begin{tabular}{lcccc}
\hline \hline Disciplina & Privada & Estatal & Significancia & Mayor \\
\hline \hline Expresión & .39 & .43 & No & Similares \\
\hline Didáctica & .57 & .62 & Si & Estatal \\
\hline Gestión & .52 & .53 & No & Similares \\
\hline Entrenamiento & .55 & .58 & No & Similares \\
\hline Deporte & .56 & .57 & No & Similares \\
\hline Recreación & .59 & .59 & No & Similares \\
\hline Salud y Primeros Auxilios & .44 & .46 & No & Similares \\
\hline \hline
\end{tabular}

Según la dependencia escolar, la tabla 4, expresa que solamente en el área de la Salud y Primeros Auxilios, las/los estudiantes pertenecientes a colegios Particulares y Subvencionados están significativamente sobre el resto. En general, en las otras áreas no hay diferencias significativas, es decir, son similares. Sin embargo, los colegios municipales y subvencionados presentan una menor dispersión respecto a los particulares pagados.

TABLA 4

Conocimientos disciplinarios según dependencia escolar

\begin{tabular}{cccccc}
\hline Disciplina & Municipal & Subvencionado & Particular & Significancia & Mayor \\
\hline Expresión & .40 & .43 & .42 & No & Similares \\
\hline Didáctica & .40 & .43 & .42 & No & Similares \\
\hline Gestión & .54 & .51 & .52 & No & Similares \\
\hline Entrenamiento & .57 & .57 & .63 & No & Similares \\
\hline Deporte & .56 & .57 & .53 & No & Similares \\
\hline Recreación & .58 & .61 & .50 & No & Similares \\
\hline $\begin{array}{c}\text { Salud y Primeros } \\
\text { Auxilios }\end{array}$ & .43 & .46 & .55 & SI & $\begin{array}{c}\text { Particular- } \\
\text { Subvencionado- } \\
\text { Municipal }\end{array}$ \\
\hline \hline
\end{tabular}

Respecto al Género la tabla 5, comunica que, en la disciplina de Expresión, los varones superan significativamente a las damas. En Entrenamiento, las damas superan a los varones significativamente. En Deporte, las damas superan a los varones significativamente. En las demás disciplinas, se observa que son similares. 
TABLA 5

Conocimientos disciplinarios según Género

\begin{tabular}{lcccc}
\hline \hline Disciplina & Damas & Varones & Significancia & Mayor \\
\hline \hline Expresión & .39 & .46 & $\mathrm{Si}$ & Varones \\
\hline Didáctica & .58 & .62 & No & Similares \\
\hline Gestión & .53 & .51 & No & Similares \\
\hline Entrenamiento & .59 & .54 & $\mathrm{Si}$ & Damas \\
\hline Deporte & .58 & .53 & $\mathrm{Si}$ & Damas \\
\hline Recreación & .60 & .58 & No & Similares \\
\hline Salud y Primeros Auxilios & .45 & .45 & No & Similares \\
\hline \hline
\end{tabular}

Finalmente, en la tabla 6 y en la figura 1, se resumen los desempeños disciplinarios, siendo U2, quien presenta mejores resultados en la mayoría de las disciplinas. Por su parte la U6, es la institución de Educación Superior más descendida respecto a conocimientos disciplinarios.

TABLA 6

Resumen de Desempeños disciplinarios

\begin{tabular}{cccccc}
\hline \hline Disciplina & Por Universidad & $\begin{array}{c}\text { Por tipo } \\
\text { Universidad }\end{array}$ & Género & Dependencia & General \\
\hline \hline Expresión & U2-U5;U4-U1-U3-U6 & Similares & Varones & Similares & 3 \\
\hline Didáctica & U2-U1;U3-U6-U4-U5 & Estatal & Similares & Similares & 1 \\
\hline Gestión & Similares & Similares & Similares & Similares & 2 \\
\hline Entrenamiento & Similares & Similares & Damas & Similares & 1 \\
\hline Deporte & Similares & Similares & Damas & Similares & 1 \\
\hline Recreación & U2-U3-U5;U4-U1-U6 & Similares & Similares & Similares & 1 \\
\hline $\begin{array}{c}\text { Salud y Primeros } \\
\text { Auxilios }\end{array}$ & U2-U5-U1;U4-U3-U6 & Similares & Similares & Particular-Subvencionado- & Municipal \\
\hline \hline General & U2 U1-U4-U5-U3-U6 & & & & \\
\hline \hline
\end{tabular}


FIGURA 1

Resumen de intervalos de confianza entre Universidades

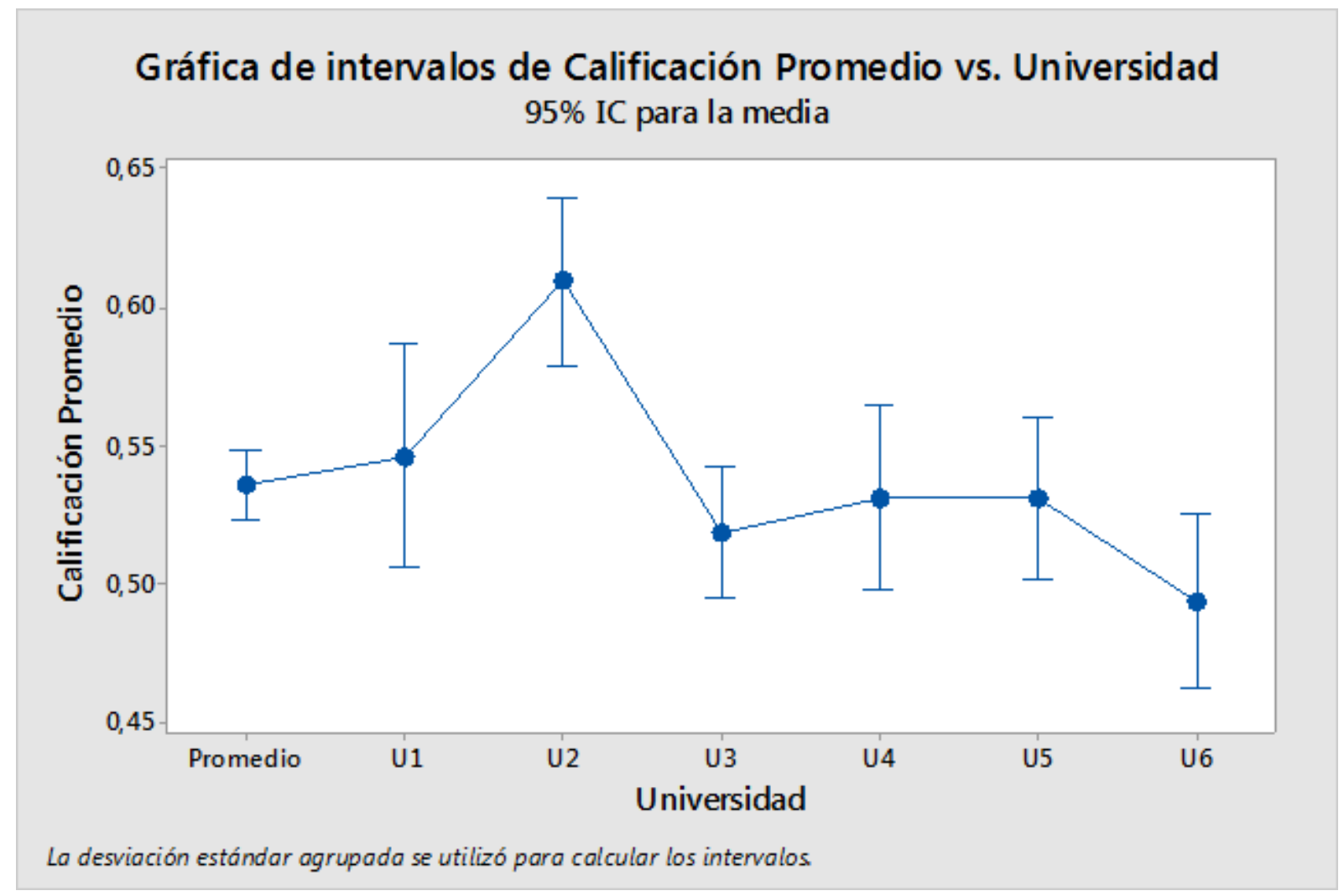

\section{Discusión}

De los resultados del estudio, se desprende que es necesario que las disciplinas y las universidades formadoras de Profesores de Educación Física, se desarrollen de manera más equilibrada y en su conjunto, puedan elevar significativamente el nivel de dominio respecto a la media. Si bien U2, obtuvo el mejor desempeño dentro del conjunto de disciplinas y universidades, sus resultados evidencian un desarrollo marginal. Lo anterior, confirma la realidad evidenciada también por González, Santana, Guarda \& Molina (2006); González, Santana, Guarda \& Molina (2007); González, Santana, Guarda \& Molina (2010), quiénes en su momento recomendaron introducir medidas paliativas al interior de las entidades de Educación Superior, antes del egreso de los estudiantes. Estas evidencias, ratifican, además, los resultados de la Prueba Inicia del 2011- 2013, donde los docentes noveles examinados de la época, obtuvieron resultados poco favorables y, por tanto, no reunían los requisitos mínimos que lo habiliten para un desempeño profesional docente. Se suma a ellos, los recientes resultados nacionales obtenidos respecto a Conocimientos Disciplinarios y Didácticos evaluados por el Centro de Perfeccionamiento, Experimentación e Investigaciones Pedagógicas del Ministerio de Educación (CPEIP - MINEDUC 2016-2017 y 2018), donde se advierten también debilidades en el manejo de conocimientos teóricos y procedimentales de las entidades de Educación Superior, formadoras de Profesores de Educación Física.

Estos resultados, son coherentes, con apreciaciones planteadas por Páez y Hurtado (2019), en el sentido de que las competencias más valoradas en el proceso formativo del profesor de Educación Física son en el ámbito del hacer y al mismo tiempo, coincide con las apreciaciones que hace Hunt (2009) en el contexto de que los rendimientos académicos de los estudiantes del FID dependen principalmente de la calidad de los docentes que imparten las distintas asignaturas, en especial las prácticas; esto haría suponer, que el éxito 
o fracaso del rendimiento académico del estudiante de Educación Física es muy dependiente del docente a cargo de una determinada asignatura (Páez y Hurtado, 2019), lo que implicaría que los discentes que presentan mayor éxito en el rendimiento de las pruebas administradas en este estudio, serían aquellos alumnos que cuentan con profesores mejor preparados, y con mayor experiencia en cada una de las áreas medidas. Es oportuno señalar también, que, en el contexto de la FID, el colectivo de estudiantes del área, transita con experiencias adquiridas durante la escolarización que influyen sustancialmente en cómo entienden y afrontan el proceso para convertirse en docentes, es decir, las experiencias vividas y las creencias construidas durante sus años previos afectan la manera que tienen de abordar su periodo universitario de formación y, también, su futuro como docentes (Gómez y Guerra, 2012). Bajo este escenario, el panorama hasta ahora no resulta muy auspicioso y el desafío, de acuerdo a Bases Curriculares de Educación Física y Salud, generadas desde el Ministerio de Educación de Chile, es que el proceso educativo no debe disputarse, debe otorgar relevancia a las evaluaciones de conocimientos teóricos como también en ámbitos procedimentales. Ambos, junto a la dimensión valórica y actitudinal le otorgan relevancia al proceso pedagógico y se constituyen en instrumentos cruciales de verificación del aprendizaje y en general, del aseguramiento de la calidad de la FID. En ese escenario, el desarrollo de competencias relacionadas con el conocimiento del contenido (saber) y el conocimiento pedagógico del contenido (saber hacer), han mostrado ser dos elementos importantes para el desempeño de la futura labor profesional del docente (Almonacid-Fierro \& Vizuete Carrizosa, 2018; Rodríguez-Gómez, Del Valle y De la Vega, 2018).

\section{Conclusiones}

A partir de los resultados, es posible determinar que los conocimientos disciplinarios de las y los estudiantes de último semestre de las carreras de Educación Física y Deporte respecto a las actuales Bases Curriculares, poseen un desempeño regular conforme a dominios de contenido vinculados a las siete disciplinas definidas previamente, pero levemente sobre el promedio en todas las áreas, independiente a la Universidad a la que pertenecían, por tanto, solo un mínimo de calidad para ejercer en perspectiva su profesión, al menos desde la dimensión de formación disciplinaria.

Se sugiere, que, en próximas indagaciones, sean estudiadas de manera más detallada, aquellos casos donde fue posible encontrar diferencias significativas y de esta forma, entender en mayor profundidad el fenómeno $\mathrm{y}$ actuar en consonancia.

Los resultados permiten aproximarse a la realidad, son de utilidad y pueden contribuir en la toma de decisiones asociadas a la política pública y planes de mejoras de las propias casas de estudios.

Se sugiere que para las próximas investigaciones se pueda ampliar el estudio a otras universidades que son parte de las entidades privadas como también, a otras entidades de Educación Superior del área Latinoamericana, lo que supondría un contraste interesante y un espacio de intercambio y de análisis de experiencias que potencien cada vez la Formación Inicial Docente de calidad y permitan resignificar el valor de la evaluación formativa.

Finalmente, los profesores en formación deben considerar la complejidad y la diversidad social y cultural e ir más allá de los contenidos del currículum escolar nacional, sin embargo, es relevante también, empoderarse respecto al conjunto de conocimientos, habilidades, actitudes y valores que las Bases Curriculares promueven, los cuales en virtud de una epistemología y realidad han sido seleccionados por el Estado.

\section{Agradecimientos}

Este trabajo se ha llevado a cabo dentro del Proyecto de Investigación FGI - 17 - liderado por la red UMCE y también del Proyecto DID - I - 2017 - 09 - UACH y Proyecto DIE 17-0008 - UFRO, denominado 
"Calidad de la formación de los alumnos en carreras de Pedagogía en Educación Física, en entidades Universitarias del país”.

\section{ReFERENCIAS}

Almonacid-Fierro, A. \& Vizuete Carrizosa, M. (2018). Validación de un cuestionario para medir el conocimiento didáctico del contenido en el profesorado de Educación Física. Retos. Nuevas Tendencias en Educación Física, Deporte y Recreación, (34), 132-137.

Ávalos, B. (2005). Las instituciones formadoras de docentes y las claves para formar buenos docentes. En R. Lara \& D. Rojas (Comp.), El desafio de formar los mejores maestros: situación actual, experiencias, innovaciones y retos en la formación de los formadores docente (pp. 14- 22). Bogotá: Universidad Pedagógica Nacional.

Bravo, D.; Falk, D.; González, R.; Manzi,J. \& Peirano, C. (2008). La relación entre la evaluación docente y el rendimiento de los alumnos: Evidencia para el caso de Chile. Santiago: Centro de Microdatos, Departamento de Economía, Universidad de Chile y Centro de Medición. Disponible en http://www.microdatos.cl/docto_publicaciones/Eva luacion\%20docentes_rendimiento\%20escolar.pdf

Caena, F. (2011). Literature review Teachers' core competences: requirements and development. Brussels: European commission. Recuperado desde http://ec.europa.eu/dgs/education_culture/repository/education/policy/strat egic-framework/doc/teacher-competences_en.pdf

Cid, Alfonso, Andrés Pérez y Miguel Zabalza (2009), "Las prácticas de enseñanza declaradas de los 'mejores profesores' de la Universidad de Vigo”. Relieve, 15(2), 1-29. Recuperado de: http://www.uv.es/RELIEVE/v15n2/RELIE VEv15n2_7.htm

Desrosiers, P., Genet-Volet, Y., \& Godbout, P. (1997). Teachers' Assessment Practices Viewed Through the Instruments Used in Physical Education Classes. Journal of Teaching in Physical Education, 16(2), 211-228.

Gaete, M. (2008). Sentido de la enseñanza de la Filosofía en la escuela secundaria. Una mirada desde los profesores guias de las prácticas profesionales (Tesis doctoral). Universidad de Barcelona, Barcelona, España.

González, M, Santana R., Guarda S. \& Molina E. (2006). Diseño y validación de Instrumentos para evaluar la Calidad $y$ Cantidad de la Educación Física en escolares de $5^{\circ}$ a $8^{\circ}$ año de Enseñanza Básica. Universidad Metropolitana de Ciencias de la Educación. Santiago, Chile.

González, M, Santana R., Guarda S., Molina E. (2007). Calidad y Cantidad de La Educación Física en Escolares de $5^{\circ}$ $A 8^{\circ}$ de Educación Básica en La Comuna de Nuñoa. Universidad Metropolitana de Ciencias de la Educación. Santiago, Chile

González, M, Santana R., Guarda S., Molina E. (2010). Evaluación y Determinación de Estándares de Calidad de la Educación Física en la Enseñanza General Básica. Universidad Metropolitana de Ciencias de la Educación. Santiago, Chile

González, M., Molina, E., Beltrán, A., Flández, J., Negrón, M., Martínez, C., Vargas, R. Trujillo, H., Ferreira, M., Leiva, R., Lobos, M. (2020). Construcción y validación de un instrumento para valorar la calidad de la formación de profesores de Educación Física en Chile. Retos. Nuevas Tendencias en Educación Física, Deporte y Recreación, 37, 312-319.

Gómez, V., \& Guerra, P. (2012). Teorías implícitas respecto a la enseñanza y el aprendizaje: ¿Existen diferencias entre profesores en ejercicio y estudiantes de pedagogía? Estudios pedagógicos (Valdivia), 38(1), 25-43.

Hativa, Nira y Peter Goodyear (2002), Teacher Thinking, Beliefs and Knowledge in Higher Education, Dordrecht/ Boston/Londres, Kluwer Academic Publishers

Herrera, J. D. C. P., \& Almonacid, J. H. (2019). Formación inicial docente en profesores de educación física. Levantamiento de competencias específicas a partir de las necesidades del medio educativo. Retos: nuevas tendencias en educación fisica, deporte y recreación, (35), 61-66.

Hidalgo, C. D., \& Venegas, V. R. (2019). Políticas de igualdad de género en la educación superior chilena/Policies of gender equality in Chilean higher education. Revista Rumbos TS. Un espacio critico para la reflexión en Ciencias Sociales, (19), 41-72. 
Hortigüela, D., Pérez-Pueyo, A. \& Fernández-Río, J. (2016). Influencia de las experiencias vivenciadas por el alumnado en el desempeño de futuras competencias docentes. Contextos Educativos, 19, 25-41. DOI: https://10.18172/ con. 2742

Hunt. B. (2009). Efectividad del desempeño docente. Una reseña de la literatura internacional y su relevancia para mejorar la educación en América Latina. Santiago, Chile:San Marino

López-Pastor, V., \& Sicilia-Camacho, A. (2017). Formative and shared assessment in higher education. Lessons learned and challenges for the future. Assessment \& Evaluation in Higher Education, 42(1), 77-97.

Machado, D. (2018). Perspectiva del concepto de calidad en la Educación Superior en América Latina. Revista Navarra Juridica, 2(1), 54-74.

Ministerio de Educación de Chile (2001). Estándares de desempeño para la formación inicial de docentes. Recuperado en diciembre 2017 de: http://www.educarchile.cl/userfiles/P0001/File/estándaresformacióndocente.pdf

Ministerio de Educación de Chile. (2013). Evaluación INICIA. Presentación de Resultados 2012. Santiago de Chile: MINEDUC.

Ministerio de Educación de Chile. (2019) Centro de Perfeccionamiento, Experimentación e Investigaciones Pedagógicas. Resultados Nacionales. Evaluación Nacional Diagnostica de la Formación Inicial Docente 2016-2017-2018. Recuperado de https://www.cpeip.cl/formacion-inicial-docente/. Fecha de acceso: septiembre 2019.

Organización de las Naciones Unidas para la Educación, la Ciencia y la Cultura (UNESCO) (2018). Women in Science. Fact Sheet No. 51 June 2018 FS/2018/SCI/51. Recuperado de: http://uis.unesco.org/sites/default/fi les/documents/fs51-women-in-science-2018-en.pdf

Páez, C. J. \& Hurtado, A. J. (2019). Formación inicial docente en profesores de educación física. Levantamiento de competencias específicas a partir de las necesidades del medio educativo. Retos. 35, 61-66.

Pedraja-Rejas, L. M., Araneda-Guirriman, C. A., Rodríguez-Ponce, E. R., \& Rodríguez-Ponce, J. J. (2012). Calidad en la formación inicial docente: evidencia empírica en las universidades chilenas. Formación universitaria, 5(4), $15-26$.

Rodríguez-Gómez, I., Del Valle, S., y De la Vega, M. (2018). Revisión nacional e internacional de las competencias profesionales de los docentes de Educación Física. Retos. Nuevas Tendencias en Educación Física y Deportes, 34, 383-388

Ruffinelli, A. (2013). La Calidad de la Formación Inicial Docente en Chile: la perspectiva de los profesores principiantes. Revista Calidad en la Educación, (39), 117-154.

Ruffinelli, A. (2014). Dificultades de la iniciación docente: eiguales para todos? Estudios Pedagógicos, 40(1), 229-242.

Sotomayor, C., \& Gysling, J. (2011). Estándares y regulación de calidad de la formación de profesores: discusión del caso chileno desde una perspectiva comparada. Calidad en la educación, (35), 91-129. 